\title{
Effect of Diabetes Duration on Proliferative Disease Regression after Panretinal Photocoagulation Using a Conventional Laser Versus Pattern Scan Multispot Laser
}

\author{
Tamer Ahmed*, Ahmed Howaidy, Abd Allah El-Amin, Khalid Selim \\ Department of Ophthalmology, Faculty of Medicine, Aswan University, Egypt \\ *Corresponding author: Tamer Ahmed Ali Ahmed; Mobile: +201006508312; Email: tamerahmedali@yahoo.com
}

\begin{abstract}
Background: Panretinal photocoagulation (PRP) is considered the gold standard for first line therapy in proliferative diabetic retinopathy (PDR). The aim of PRP is to prevent visual loss following vitreous hemorrhage, tractional retinal detachment, and neovascular glaucoma, by leading to regression of neovascularization.

Objective: The purpose of this study was to compare the effect of diabetes duration on proliferative disease regression after pan-retinal photocoagulation using a conventional green laser versus a multispot pattern scan laser. Subjects and Methods: Eighty eyes with newly diagnosed proliferative diabetic retinopathy were randomly divided in to two groups each composed of 40 eyes: group (A) in which patients were received standard argon laser panretinal photocoagulation and group (B) in which patients were received pattern scan multispot panretinal photocoagulation. Changes in central macular thickness and BCVA at 1st, 4th, 12th week and 6th month follow-up were compared to baseline measurements. Fundus fluorescein angiography was performed at 4 weeks, 12 weeks and 6 months to assess regression of PDR.

Results: by the end of the study, complete regression occurred in 37 (92.5\%) of patients in conventional laser group and $36(90 \%)$ patients in PASCAL group, while persistence of the disease occurred in $3(7.5 \%)$ patients in each group and recurrence occurred 1 (2.5\%) in PASCAL group. There was no statistically significant correlation in each study group between clinical outcome and age or duration of DM.

Conclusion: There was no statistically significant correlation in each study group between duration of DM and proliferative disease regression.
\end{abstract}

Keywords: proliferative diabetic retinopathy, panretinal photocoagulation, pattern scan Multispot Panretinal Photocoagulation, Conventional argone laser.

\section{INTRODUCTION}

Diabetes mellitus (DM) is a major emerging clinical and public health problem in Egypt with a prevalence of $5-10 \%$ in the 1990s. It has been estimated that by the year 2025, nearly 9 million Egyptians (over $13 \%$ of the population over 20 years of age) will have $\mathrm{DM}^{(\mathbf{1}, \mathbf{2})}$. Epidemiological studies of DM and diabetic retinopathy (DR) in Middle Eastern countries including Egypt have shown a substantial heterogeneity in the reported prevalence of DM (3.4$29 \%)$ and its complications especially DR (7.6-60\%), even within the same country ${ }^{(3,4)}$.

The prevalence of DR in Egypt was found to be $20.5 \%$. Most patients $(82 \%)$ were not aware of the hazards of diabetes mellitus for the eyes. The prevalence of DR was statistically significantly higher in females (22 vs.17\%, p < 0.05), with longer diabetes disease duration $(\mathrm{p}<0.001)$, hypertension $(\mathrm{p}$ $<0.001)$ and absence of hypertension control ( $\mathrm{p}<$ 0.001 ), especially proliferative DR. Increasing age and poor glycemic control were associated with a nonsignificant increase in the rate of DR $(p=0.340$ and $\mathrm{p}=0.444$, respectively) ${ }^{(5)}$.

There is a strong positive relationship between the duration of diabetes and prevalence and progression of DR. Almost all type I diabetic patients and over three fourths of type II diabetic patients will have some form of DR after 20 years of the disease ${ }^{(6)}$.

Since the Early Treatment Diabetic Retinopathy Research Study, panretinal photocoagulation have been the standard of care for treating patients with diabetic retinopathy. The first lasers were argon and krypton gas in blue-green wavelengths. Their use was limited because they had short wavelengths, causing light scatter, using high levels of energy inducing photochemical damage ${ }^{(\mathbf{1})}$.

Tunable dye lasers were the next development in laser technology, maintaining the tunable lasers, however, was expensive and difficult. Further research resulted in the introduction of infrared $(810 \mathrm{~nm})$ and green $(532 \mathrm{~nm})$ wavelengths. The benefits of these lasers include reduced scatter, ease of delivery in dense ocular media, lower cost, and reduced maintenance ${ }^{(7)}$. Further development of solid-state technology brought about the production of 577-nm wavelength lasers ${ }^{(8)}$.

The Pattern Scan Multispot Laser uses a proprietary, semiautomated pattern generation technique that allows rapid delivery of laser pulses, with durations of $10 \mathrm{~ms}$ to $20 \mathrm{~ms}$ at each spot, as opposed to $100 \mathrm{~ms}$ to $200 \mathrm{~ms}$ with conventional laser (9). 
The purpose of this study was to compare the effect of diabetes duration on proliferative disease regression after pan-retinal photocoagulation using a conventional green laser versus a multispot pattern scan laser

\section{PATIENTS AND METHODS}

This prospective randomized clinical trial study included eighty eyes with PDR eighty eyes of 60 patients, 20 patients had both eyes done. They were newly diagnosed as proliferative diabetic retinopathy (PDR). Patients were recruited from Aswan Ophthalmology Hospital Outpatient Clinic. This study was conducted between January 2017, to June 2019.

\section{Ethical approval}

Approval of the Ethical and Technical Review Committee of Aswan Faculty of Medicine was obtained. Informed written consents were taken from all patients for the specific procedure.

The included subjects were randomly divided into two groups each composed of 40 eyes; Group (A) in which patients received standard argon laser panretinal photocoagulation using single spots and Group (B) in which patients received pattern scan multispot panretinal photocoagulation.

After randomization, group A were treated in Aswan eye \& laser center and group B were treated in Aswan university hospital.

Information was collected on age, sex, indication, pre-and post-laser procedure, best corrected visual acuities (BCVA) as well as outcome and complications of treatment and intra- and postprocedure pain sensation.

\section{Inclusion criteria:}

Patients with type I or type II diabetes mellitus who were newly diagnosed as proliferative diabetic retinopathy were enrolled if they met the following criteria:

1. Patients older than 18 years of age.

2. Patients with Snellen best corrected visual acuity of $6 / 60$ or better.

3. Adequate pupil dilatation and clear media to perform laser photocoagulation, digital photography and optical coherence tomography scans.

\section{Exclusion criteria:}

1. Patients with previous laser photocoagulation or macular laser treatment perior to the study eye.

2. Patients underwent recent intra-ocular surgeries within the last three months prior to the procedure.
3. Patients with media opacities (e.g. corneal opacity, cataract, vitreous hemorrhage) that interfere with the proper evaluation of the posterior segment.

4. Mean Central macular thickness area more than $300 \mu$ as measured by optical coherence tomography scans.

5. Patients who are contraindicated to fluorescein angiography (pregnancy, allergy to fluorescein dye, renal failure).

6. Patients with poor glycaemic control, glycated haemoglobin $(\mathrm{HbA} 1 \mathrm{c})$ greater than $10.0 \mathrm{mg} / \mathrm{dL}$.

7. Patients with uncontrolled hypertension, blood pressure greater or equal to $180 / 110 \mathrm{mmHg}$.

8. Patients with vitreo-retinal traction.

9. Patients who are planned for intra-ocular surgery within six month from the start of the treatment.

Chosen patients were subjected to the following:

(1) Counseling the patient about the procedure and the possible complications of panretinal laser photocoagulation; (2) Detailed general and ocular history; (3) Full ophthalmological examination including best corrected visual acuity, slit-lamb examination, IOP measurement using Goldmann applanation tonometer, and dilated fundus biomicroscopy;(4)Baseline fundus fluorescein angiography; (5) Baseline optical coherence tomography to measure central macular thickness

\section{Treatment parameters}

The pupils were dilated using $1 \%$ tropicamide and Cyclophrine (Cyclopentolate $\mathrm{HCl} 50 \mathrm{mg}+$ Phenylephrine $\mathrm{HCl} 500 \mathrm{mg}$ ) drops, and used $0.5 \%$ proparacaine-hydrochloride drops is used as topical anesthetic before the procedure. Mainster wide-field lense were used for pan retinal photocoagulation. Treatment parameters including use of a pattern or single spot, type of pattern, power, burn duration, spot size and number of burns per session were noted. Prior to starting treatment, the operator chose whether or not to do Pascal based on the random distribution after informed consents from all patients. Eighty eyes with PDR were included in this clinical trial divided into two groups:

Group (A): patients were treated with pan laser photocoagulation for PDR using conventional laser photocoagulation (Ellex Medical Pty Ltd. Integre Pro) which is a $532 \mathrm{~nm}$ green-light Diode Solid State Photocoagulator laser.

Group (B): patients were treated with PRP for PDR with pattern scan multisport photocoagulation using a 532nm laser with computer-guided scanning technology (PASCAL Streamline Photocoagulator, Topcon Medical Laser Systems). 


\section{Laser technique}

The PASCAL PRP parameters were defined as 200 $\mu \mathrm{m}$ spot size, $20 \mathrm{~ms}$ pulse duration, and power was adjusted until a gray-white lesion was observed starting from $200 \mathrm{~mW}$. The whole PRP treatment was performed in two sessions, for PRP, the $3 \times 3,4 \times 4$ and $5 \times 5$ arrays were most commonly used. Pattern array near-simultaneously setting was used with a single depression of the foot switch. All burns were placed one burn width apart. Conventional laser PRP parameters were defined as $200 \mu \mathrm{m}$ spot size, $100 \mathrm{~ms}$ pulse duration, and power increased from $200 \mathrm{~mW}$ until a gray-white lesion was attained. Burns were placed one burn width apart. All of the patients completed the entire PRP treatment in two or three sessions. Burn distribution was greater than 2-disc diameters (DD) temporal to the fovea, no closer than one row within the arcades, and burn placement as close to the ora serrata as possible.

Pain score: A mean value between numerical rating pain score and The Wong-Baker Faces Pain Rating Scale was used to record the pain score.

\section{Data extraction strategy}

Data were extracted into a predesigned data extraction form. Information collected included age, sex, DM duaration, procedure, best-corrected visual acuity (BCVA), clinical efficacy and outcome, and complications following laser. Data were also collected on the parameters used for the treatment including power, pulse duration, number of burns per treatment session and retinal spot size.

\section{Statistical analysis}

The data was analyzed using IBM SPSS Statistics 24.0 program. Screening for extreme values in quantitative variables was done using independent ttest. Discrete and categorical variables were screened using frequency distribution, Chi-square test $\&$ Fisher Exact Test. Correlation with Pearson correlation. Visual acuities (VA) were converted from Snellen to $\log$ Mar to explore changes in vision pre- to postlaser. $p$ value of $<0.05$ was considered significant and highly significant $(\mathrm{p}<0.001)$.

\section{RESULTS}

Eighty eyes of 60 patients, 20 patients had both eyes done, were included in this clinical trial; of whom $26(43.33 \%)$ were male, and $34(56.67 \%)$ were female with a mean age of 54.47 years (SD 8.72, range 31 - 75). There were 40 eyes in the conventional laser group (A) and 40 eyes in the PASCAL group (B).

For eyes in group A, the mean age of the patients was 52.8 (SD 8.78, range 31 - 69) of whom 18 (45\%) were for males and $22(55 \%)$ were for females. For patients in group $\mathrm{B}$, the mean age of the patients was 56.14 (SD 9.55, range 35 - 75) of whom 17 (42.5\%) were for males and 23(57.5\%) were for females.

At baseline (table 1), there was no significant difference between the groups ( $p>0.05$, for all) in terms of age, sex, most recent glycated hemoglobin, DM duration, BCVA, or CMT. For patients in group $\mathrm{A}$, the mean DM duration was $15 \pm 9.75$ (range 5 25 ), for patients in group $\mathrm{B}$, the mean DM duration was $15 \pm 9.25$ (range $5-30$ ).

Table (1): Comparison between the two studied groups regarding the demographic data and baseline parameters.

\begin{tabular}{|l|l|l|l|l|l|}
\hline Variables & \multicolumn{2}{|l|}{ Group A } & \multicolumn{2}{l|}{ Group B } & $\begin{array}{l}\text { P- } \\
\text { value }\end{array}$ \\
\hline Age & \multicolumn{2}{|l|}{$52.8 \pm$ SD 8.78 } & \multicolumn{2}{|l|}{$56.14 \pm$ SD 9.55 } & 0.326 \\
\hline Sex & Male & $\begin{array}{l}18 \\
(45 \%)\end{array}$ & Male & $\begin{array}{l}17 \\
(42.5 \%)\end{array}$ & 0.823 \\
\cline { 2 - 5 } & Female & $\begin{array}{l}22 \\
(55 \%)\end{array}$ & Female & $\begin{array}{l}23 \\
(57.5 \%)\end{array}$ & 0.529 \\
\hline $\begin{array}{l}\text { DM } \\
\text { duration }\end{array}$ & $15 \pm 9.75$ & $15 \pm 9.25$ & 0.824 \\
\hline $\begin{array}{l}\text { Baseline } \\
\text { HA1C }\end{array}$ & $7.8 \pm 1.75$ & $7.95 \pm 1.95$ & 0.596 \\
\hline $\begin{array}{l}\text { Baseline } \\
\text { BCVA }\end{array}$ & $0.3 \pm 0.22$ & $0.3 \pm 0.21$ & 0.725 \\
\hline $\begin{array}{l}\text { Baseline } \\
\text { CMT }\end{array}$ & $250 \pm 50$ & $24.6 \pm 45.25$ & \\
\hline
\end{tabular}

Regarding laser parameters for the PASCAL group the mean power of PRP used was $525 \mathrm{~mW}$ (SD 125.4, range 350 - 950) and the mean number of spots used were 2820.63 (SD 394.18, range 2200 - 3700). All procedures were done in 2 sessions. For the conventional group the mean power of PRP used was $260 \mathrm{~mW}$ (SD 130.7, range 160 - 590) and the mean number of spots used were 2611.42 (SD 285.61, range 1782 - 3121). The majority of cases were done in 2 sessions and only in 5 cases the procedure was done in 3 sessions.

Regarding the correlation of demographic data and proliferative disease regression in both groups as shown in (table 2), there was no statistically significant correlation in each study group between clinical outcome and age or duration of DM.

Table (2): Correlation between demographic data and clinical outcome throughout the study period in each group.

\begin{tabular}{|l|l|l|l|l|l|}
\hline \multicolumn{2}{|c|}{} & \multicolumn{2}{l|}{$\begin{array}{l}\text { Conventional } \\
\text { group }\end{array}$} & \multicolumn{2}{l|}{$\begin{array}{l}\text { PASCAL } \\
\text { group }\end{array}$} \\
\cline { 3 - 6 } & r & $\begin{array}{l}\text { P- } \\
\text { value }\end{array}$ & r & $\begin{array}{l}\text { P- } \\
\text { value }\end{array}$ \\
\hline \multirow{2}{*}{ Age } & $<50 \mathrm{y}$ & 0.098 & 0.428 & -0.060 & 0.580 \\
\cline { 2 - 6 } & $\geq 50 \mathrm{y}$ & & & & \\
\hline
\end{tabular}




\begin{tabular}{|l|l|l|l|l|}
\hline $\begin{array}{l}\text { Duration of } \\
\text { DM }\end{array}$ & -0.162 & 0.410 & -0.199 & 0.220 \\
\hline
\end{tabular}

- $r=$ pearson correlation

\section{DISCUSSION}

Panretinal photocoagulation (PRP) is considered the gold standard for first line therapy in proliferative diabetic retinopathy (PDR) ${ }^{(\mathbf{1})}$.

In our clinical trial, we prospectively investigated the clinical efficacy of a conventional panretinal photocoagulation versus PASCAL retinal photocoagulation in the treatment of PDR regarding DM duration. Eighty eyes with PDR were enrolled in our clinical trial; there were 40 eyes treated with conventional laser (group A) and 40 eyes treated with PASCAL laser (group B).

The mean power used in our present study was significantly higher with the Pascal system $525 \mathrm{~mW}$ (SD 125.4, range 350 - 950) than with the conventional system $260 \mathrm{~mW}$ (SD 130.7, range 160 590) ( $\mathrm{p}$ 0.001). So higher power settings were needed with the Pascal system as compared with conventional photocoagulation.

However, these higher power levels required with the Pascal system did not result in any serious complications. This may be a reflection of the reduced laser energy per burn reaching the eye secondary to its shorter duration. Fluence is calculated as (power xtime/area), and provided that spot size remains unchanged, with a burn duration of $20 \mathrm{~ms}$ the fluence is less than with a $100 \mathrm{~ms}$ burn when titrating to the same burn intensity because of reduced diffusion of heat ${ }^{(\mathbf{1 0})}$.

Another study Blumenkranz et al. (11) has demonstrated that $20 \mathrm{~ms}$ pulse durations represent an optimal compromise between reduced collateral damage and sufficient width of the therapeutic window ${ }^{(12)}$.

The total number of laser spots applied to each eye was calculated for each group. In the conventional group, the mean number of PRP burns used was 2611.42 (SD 285.61, range 1782-3121) delivered on average over two sessions (range $2-3$ sessions), and this was significantly lower than the PASCAL group 2820.63 (SD 394.18, range 2200 - 3700) delivered over two sessions, $(\mathrm{P}=0.03)$.

Laser parameters regarding power, spot size and number of burns that has been used in our present study was comparable to many studies like Salamn et al. ${ }^{(13)}$, Nagpal et al. ${ }^{(14)}$, Muqit et al. ${ }^{(15)}$ and Muraly et al. ${ }^{(16)}$.

In our study both groups showed favorable success rates. After 3 months complete regression occurred in $33(82.5 \%)$ of patients in conventional laser group and $30(75 \%)$ patients in PASCAL group, reduction of $\mathrm{NV}$ occurred in $5(12.5 \%)$ patients in conventional group and 3 (7.5) in the PASCAL group, while persistence of the disease occurred in $2(5 \%)$ patients in conventional laser group and 7 (17.5\%) in PASCAL group. More laser photocoagulation was need for patients who developed partial regression or persistence of PDR.

The average power with the conventional photocoagulator for these 7 procedures was $346 \mathrm{~mW}$ (135.6 SD, range $250-590$ ), and the mean number of burns was 725 (SD 235.2, range 410-850). The Pascal parameters used for these 10 procedures were as follows: mean power was $500 \mathrm{~mW}$ (150.2 SD, range $350-800$ ), and the mean number of burns was 850 (SD 290.18, range 550-1400).

After 6 months complete regression occurred in $37(92.5 \%)$ of patients in conventional laser group and $36(90 \%)$ patients in PASCAL group while persistence of the disease occurred in $3(7.5 \%)$ patients in each group and recurrence occurred in 1 $(5 \%)$ in PASCAL group.

In a study by Muraly et al. (16) comparing PASCAL and $532 \mathrm{~nm}$ conventional laser, one eye of each patient was treated with single session PRP using the PASCAL system (mean 2,795 spots), while the other eye was treated with multi-sessions PRP using a conventional laser over 2 or 3 sessions (mean 1,414 spots). PASCAL PRP was $90 \%$ effective and conventional PRP was 64\% effective at 1 month; both were $98 \%$ effective at 6 months.

In the Manchester Pascal Study, Muqit et al. ${ }^{(15)}$ studied 40 eyes of 24 patients with PDR. Half of the eyes were treated with 1,500 single spots at $100 \mathrm{~ms}$ duration using PASCAL over the course of 3 sessions at 2-week intervals; the other eyes were treated with 1,500 spots in a single session of 20 -ms multispot laser. Twelve weeks after treatment, single session PRP was $74 \%$ effective and multi-session PRP was $53 \%$ effective, although the difference was not statistically significant.

In this study regarding the correlation of diabetes duration and proliferative disease regression there was no statistically significant correlation in each study group between clinical outcome and age or duration of DM.

\section{CONCLUSION}

There is no effect of diabetes duration on proliferative disease regression after pan-retinal photocoagulation using a conventional laser versus pattern scan multispot laser

\section{REFERENCES}

1. Arab M (1992): Diabetes mellitus in Egypt. World Health Stat Q., 45: 334-337. 
2. Herman WH, Ali MA, Aubert RE et al. (1995): Diabetes mellitus in Egypt: risk factors and prevalence. Diabet Med., 12: 1126-1131.

3. Alwan A, King $H$ (1992): Diabetes in the eastern Mediterranean region. World Health Stat Q., 45: 355359.

4. El-Shazly M, Zeid M, Osman A (2000): Risk factors for eye complications in patients with diabetes mellitus: development and progression. East Mediterr Health J., 6: 313-325.

5. Macky TA, Khater N, Al-Zamil MA et al. (2011): Epidemiology of diabetic retinopathy in Egypt: a hospital-based study. Ophthalmic Res., 45 (2): 73-78.

6. American Diabetes Association (2010): Standards of medical care in diabetes-2010. Diabetes Care, 33(1):11-61.

7. Nagpal M, Marlecha $S$ and Nagpal $K$ (2010): Comparison of laser photocoagulation for diabetic retinopathy using 532-nm standard laser versus multispot pattern scan laser. Retina, 30(3):452-458.

8. Kent C (2007): Laser Evolution: True Yellow Meets Solid State. Review of Ophthalmology, 7(24): 36-42.

9. Modi D, Chiranand $P$ and Akduman $L$ (2009): Efficacy of patterned scan laser in treatment of macular edema and retinal neovascularization.Clin Ophthalmol., 3:465-470.
10. Bailey CC, Sparrow JM, Grey RH et al. (1999): The National Diabetic Retinopathy Laser Treatment Audit. III. Clinical outcomes. Eye, 13: 151-159.

11. Blumenkranz MS, Yellachich D, Andersen DE et al. (2006): Semiautomated patterned scanning laser for retinal photocoagulation. Retina, 26(3):370-376.

12. Meyer-Schwickerath G (1956): Prophylactic treatment of retinal detachment by light coagulation. Trans Ophthalmol Soc UK., 76:739-750.

13. Salman A (2011): Pascal laser versus conventional laser for treatment of diabetic retinopathy. Saudi J Ophthalmol., 25(2):175-179.

14. Nagpal M, Marlecha S, Nagpal $K$ (2010): Comparison of laser photocoagulation for diabetic retinopathy using 532-nm standard laser versus multisport pattern scan laser. Retina, 30(3):452-458.

15. Muqit MMK, Marcellino GR, Henson DB et al. (2010): Single-session vs multiple-session pattern scanning laser panretinal photocoagulation in proliferative diabetic retinopathy. Arch Ophthalmol., 128(5): 525-533.

16. Muraly P, Limbad P, Srinivasan K et al. (2011): Single session of pascal versus multiple sessions of conventional laser for panretinal photocoagulation in proliferative diabetic retinopathy. A Comparitive Study. Retina, 31:1359-1365. 